\title{
New perspectives for optical techniques in diagnostic and treatment of hepatic diseases ${ }^{1,2}$
}

\author{
Novas perspectivas para técnicas ópticas no diagnóstico e tratamento de doenças hepáticas
}

\author{
Vanderlei Salvador Bagnato ${ }^{I}$, Cristina Kurachi' ${ }^{I I}$, Orlando de Castro-e-Silva ${ }^{\text {III }}$ \\ ${ }^{\text {I }}$ Full Professor and Head of the Optics and Photonics Center, Institute of Physics Institute of São Carlos of University of São Paulo, Brazil. \\ ${ }^{\text {II }} \mathrm{PhD}$, Professor of Institute of Physics of University of São Paulo, Brazil.

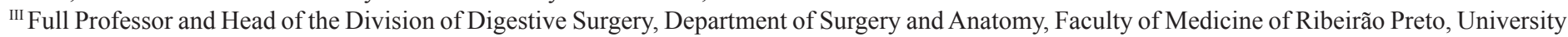 \\ of São Paulo, Brazil. \\ ${ }^{1}$ Institute of Physics Institute of São Carlos of University of São Paulo, Brazil. \\ ${ }^{2}$ Faculty of Medicine of Ribeirão Preto, University of São Paulo, Brazil.
}

\section{Introduction}

Human history is filled with great examples where light has played an important role in medicine. This idea has been considered more seriously in recent years, originating a new field that combines health science, physics and engineering. This connection is presently defined as biophotonics. The recent burst of activity experienced by this field is a result of a series of developments achieved in the last few years. The first important milestone in the development of biophotonics was transformation of light sources, like lasers and LEDs, in accessible tools for research and applications. Lasers, in special, are now broadly used as excitation light sources for disease diagnostics and as optical scalpels in interventional surgery. Another important aspect of the recent valuable progress is the advance of photochemistry applied to molecules of biological interest. Today, biophotonics can bring new technologies to define a bright future to biomedical research and clinical applications. This important advance comes in a good time, since the present growing and seniority of our modern society is demanding the development of new possibilities, that within the economical reality, make treatment, diagnostics and prevention techniques available to everyone. Biophotonics is certainly a source of solutions in this aspect.

\section{Purpose}

The purpose of this contribution is to highlight recent development that indicates modern applications for the treatment and diagnostics of hepatic diseases. In special, we would like to focus the attention to the tools and principles that compose the optical diagnostics of hepatic related disease. Application to evaluate liver steatosis and liver graft viability for transplantation are given indications of optical diagnostics becoming a great reality for clinical use.

In a recent article published by our group ${ }^{1}$ the use and the potentiality for optical biopsy to evaluate the degree of steatosis in a liver animal model was demonstrated. Almost simultaneously, another article demonstrated the possibility to evaluate liver drafts has also impacted the community. That result has called the attention of many international groups, and the article ends generating a special editorial about the subject on the same journal. This is certainly a nice starting point to introduce the possibilities of such techniques for clinical applications.

\section{Techniques}

In brief, spectroscopic signals can indicate biochemical changes, and these generally precede the morphological changes observed in histology. Most important, however, is the fact that the information provided by spectroscopy is quantitative, and thus it can bring an added degree of objectivity to the process of diagnosing disease.

Several spectroscopic techniques can be used to diagnose tissue: reflectance, fluorescence, infrared absorption, Raman spectroscopy among others ${ }^{2-4}$. Each of these has its own special features and potential applications. Laser-induced Fluorescence (LIF) has been shown to be a very sensitive analytical technique for biochemical analysis at ultratrace levels. This technique has also been demonstrated to be a useful tool for monitoring DNA damage and for cancer diagnostics.

In the article presented in reference ${ }^{1}$, the fluorescence spectroscopy system is described. Basically the system is composed of: i) One spectrometer, which goes from $350 \mathrm{~nm}$ up to $850 \mathrm{~nm}$; ii) One Y-shaped fiber, which delivers the laser light through one central fiber and collects the fluorescence from the tissue using six periferical fibers; iii) And an excitation source, emitting at $532 \mathrm{~nm}$ (doubled-frequency Nd:YAG). The laser power is in the order of 5 $\mathrm{mW}$, assuring no thermal effect on the incident spot. It should be pointed out that the signal from the backscattering (emission at the same wavelength of excitation light) is about thousand times more intense than the one from the broad fluorescence band. To simplify the analyses we used an optical filter to reduce it one thousand times; this way both parts of the spectrum present comparable intensities. The spectrum was collected by direct contact between the fiber and the liver. To protect the fiber and to avoid contamination from one measurement to the other, we used a 
non-fluorescent transparent plastic film between the liver tissue and the fiber. Once the fluorescence spectrum is obtained, a mathematical procedure is performed, allowing the verification of modifications in the spectrum, depending on tissue characteristics as steatosis degree or other tissue modifications.

\section{Samples}

The investigated animals were divided into groups according to the induced steatosis, which were determined through histological exam. Fat concentration in the liver was evaluated using lipid extraction techniques well demonstrated in the literature.

The excitation frequency is represented by the sharp observed peak. Thereafter, the broad band extended from the excitation to longer wavelengths is fully associated with the fluorescence emitted by the tissue. Because the quantity of fat interacts differently with the light as compared with the healthy tissue, the fluorescence, peak decrease the overall amplitude. Interesting fact is that this decrease in fluorescence amplitude and a determined Steatosis Fluorescence Factor (SFF) is directly correlated with the quantity of fat in the tissue.

The existence of a good correlation allows the determination of a mathematical relation to determine the degree of steatosis once the amplitude of fluorescence is determined. Once the process is fully understood, the necessity of obtain the full spectrum is no longer necessary. Just the reading of a single fluorescence wavelength allows this determination. During excitation, there is a collection of molecules that contributes to the broad emission. Coenzymes such as flavins, proteins and acid arachidonic, presented in the liver may be the main contributors for the observed fluorescence. In special, riboflavin seems to be connected with the mitochondrial activity, which may well be the responsible for the fluorescence decrease as the quantity of fat increases. Another important component is the lipofuscin, which presence in the autofluorescence of health tissue is dominant, with remarkable modification during the occurrence of steatosis. This biochemistry and many others, result in decreasing the overall fluorescence as the degree of steatosis becomes severe.

This demonstration of "proof of principles" using an animal model, certainly open up a great deal of possibilities for clinical use of the technique as well as on the experimental part. Starting by the experimental part, because the sampling is local and can even be done in the interior of the organ by fiber insertion, one will be able to understand spatial distribution and development of steatosis. That will provide enough insights for a deep understanding of this process. The understating of animal models will certainly contribute for the present clinical initiatives for patients with high degree of steatosis. On the clinical site, there seems to be many advantages for the use of fluorescence when compared to conventional biopsy. The fact that this analysis can be performed in real time, open many opportunities for clinical decision during the surgical procedure, as well as during the organ collection for transplantation, avoiding long waiting for biopsy result. Since steatosis is an important factor related to the success of liver transplantion, the inclusion of fluorescence detection during the graft evaluation, part of the liver transplantation procedure, will constitute a good auxiliary tool.

Besides the evaluation of steatosis, a recent published work, demonstrated experimentally, the applicability of LIF in evaluating the viability of rat liver previously perfused with saline solution ${ }^{5}$. Mitochondrial function and cellular ATP content of the liver were used as indicators of its metabolic status. The spectroscopic findings were then compared on a time scale with the mitochondrial function. Therefore, the aim of the study was to verify LIF's capability to detect changes taking place in the organ. Structural changes usually occur at later stages and frequently denote irreversible damage.

The satisfactory results obtained in the animal studies of liver steatosis ${ }^{1}$ and liver monitoring after perfusion ${ }^{5}$ motivated the clinical study where the potentiality of the fluorescence spectroscopy for liver graft evaluation has been investigated in transplantation patients. The preliminary results were recently published ${ }^{6}$.

Each spectrum has a common peak centered at the excitation wavelength followed by a broad emission band at longer wavelengths. This broad band of fluorescence has various contributions. We believe that many endogenous chromophores contribute to this band. The fluorescence of reduced nicotinamide adenine dinucleotide (NADH) may have special contribution as it is an essential part of the cellular energy metabolism, and it could therefore reveal the energy status of the cell. Although isolated NADH does not show prominent fluorescence under green $(532 \mathrm{~nm})$ excitation, in the intact organ (hepatocellular medium), NADH fluorescence may behave in a significantly altered manner, to the point of responding to green excitation. This, however, remains to be investigated. In order to obtain better organ preservation and consequently increase the donor pool, there is a lot still to be learned about the metabolism after organ perfusion and removal as well as about how individual chromophores in a cellular environment contribute to the fluorescence spectrum. The results show that fluorescence spectroscopy is potentially a powerful tool to be employed in evaluating the level of tissue integrity. This could be of great value in liver transplantation by providing means for evaluating the organ to be implanted. Not only could this technique offer information on the organ viability, through a noninvasive procedure and with real-time response, it can also give a quantitative result. This is of relevance as it would remove the subjectivity in the surgeon evaluation without increasing the time needed for analysis. The results also indicated that fluorescence may detect the appearance of new substances resulting from the decomposition and/or modification of the cellular structures, which could be important in analyzing the integrity of the tissue structure and function. Much remains to be done in order to turn these promises into reality in the surgical room.

The ideal imagined situation where nondestructive optical techniques can be used in biomedical diagnostics seems to be increasingly becoming reality. The analytical capabilities of the fluorescence technique introduced by laser coupled to fibers, make this tool very attractive for use together with laparoscopy or in interstitial organ analysis. Special experiments for inside organ analyses by the introduction of fibers are presently ongoing in our laboratories. That will create a procedure that goes beyond conventional biopsy. In special, for liver, is coming the time that not only light will be used to diagnostics, but also to treat diseases. Photodynamic therapy for hepatocarcinoma is becoming also a reality.

Scientists related to medical areas shall consider the possibility to experience within this field which is promising and full of new possible applications. 


\section{References}

1. Oliveira GR, Sankarankutty AK, Castro-e-Silva Jr. O, Ferreira J, Kurachi C, Zucoloto S, Vannuchi H, Jordão Jr AA, Marchini JS, Bagnato VS. Fluorescence spectroscopy to diagnose hepatic steatosis in a rat model of fatty liver. Liver Int. 2009;29:331-6.

2. Schwarz RA, Gao W, Weber CR, Kurachi C, Lee JJ, El-Naggar AK, Richards-Kortum R, Gillenwater AM. Noninvasive evaluation of oral lesions using depth-sensitive optical spectroscopy. Cancer. 2009;115:1669-79.

3. Croce AC, Ferrigno A, Vairetti N, Bertone R, Freitas I, Botiroli G. Autofluorescence spectroscopy of rat liver during experimental transplantation procedure. An approach for hepatic metabolism assessment. Photochem Photobiol Sci. 2005;4:583-90.

4. Ramanujam N. Fluorescence spectroscopy of neoplastic and nonneoplastic tissue. Neoplasia. 2000;2:89-117.

5. Sankarankutty AK, Castro-e-Silva Jr O, Ferreira J, Souza ME, Gomes MC, Kurachi C, Bagnato VS. Use of auto-fluorescence for evaluating liver grafts. Laser Phys Lett. 2003;3:539-45.

6. Castro-e-Silva O, Sankarankutty AK, Correa RB, Ferreira J, Vollet Filho JD, Kurachi C, Bagnato VS. Autofluorescence spectroscopy in liver transplantation: preliminary results from a pilot clinical study. Transplantation Proc. 2008;40:722-5.

\section{Correspondence:}

Orlando de Castro e Silva Jr

Rua Campos Sales, 890/92

14015-110 Ribeirão Preto - SP Brazil

orlando@,fmrp.usp.br

\section{How to cite this article}

Bagnato VS, Kurachi C, Castro-e-Silva O. New perspectives for optical techniques in diagnostic and treatment of hepatic diseases. Acta Cir Bras. [serial on the Internet] 2010 Mar-Apr;25(2). Available from URL: http://www.scielo.br/acb 